\title{
On an Alternative to Four Notable Distribution Functions with Applications in Engineering and the Business Sciences
}

\author{
József Dombi ${ }^{1}$, Tamás Jónás ${ }^{2}$ \\ ${ }^{1}$ Department of Computer Algorithms and Artificial Intelligence \\ University of Szeged \\ Árpád tér 2, H-6720 Szeged, Hungary \\ dombi@inf.u-szeged.hu \\ ${ }^{2}$ Institute of Business Economics \\ Eötvös Loránd University \\ Egyetem tér 1-3, H-1053 Budapest, Hungary \\ jonas@gti.elte.hu
}

\begin{abstract}
A new parametric probability distribution function is introduced and its connections with some well-known distribution functions are discussed. Due to its flexibility, we call the novel distribution function the pliant distribution function. We show that the asymptotic pliant probability distribution function can coincide with the Weibull-, exponential and logistic probability distribution functions. Furthermore, we demonstrate that with appropriate parameter settings, the novel distribution gives a simple and accurate approximation to the standard normal probability distribution. Next, we show that the pliant probability distribution function, as an alternative to the Weibull-and exponential distribution functions, can be used to model constant, monotonic and bathtub-shaped hazard functions in reliability theory. We also point out that a function transformed from the new probability distribution function can be applied in the so-called kappa regression analysis method, which may be viewed as an alternative to logistic regression.
\end{abstract}

Keywords: pliant distribution; Weibull distribution; logistic distribution; normal distribution; approximations

\section{Introduction}

In this article, a new four-parameter probability distribution function is introduced and some of its applications are discussed. As the novel distribution function is so flexible that it may be viewed as an alternative to familiar distribution functions, we call it the pliant distribution function. The cumulative distribution function of 
the novel probability distribution is based on the so-called omega function. We will demonstrate that both the omega and the exponential function $f(x)=\exp \left(\alpha x^{\beta}\right)$ $(x, \alpha, \beta \in \mathbb{R}, \beta>0)$ may be deduced from a common differential equation that we call the generalized exponential differential equation. Furthermore, we will show that the omega function, which has the $\alpha, \beta$ and $d$ parameters $(\alpha, \beta, d \in \mathbb{R}, \beta, d>$ 0 ), is asymptotically identical with the exponential function $f(x)=\exp \left(\alpha x^{\beta}\right)$. Exploiting this result, we will discuss in detail how the pliant probability distribution function can be utilized to approximate some remarkable probability distribution functions, whose formulas include exponential terms. Namely, we will show that the asymptotic pliant distribution function can coincide with the Weibull-, exponential and logistic probability distribution functions. Moreover, we will demonstrate that with appropriate parameter settings, the pliant probability distribution function can approximate the standard normal probability distribution function quite well, while the approximating formula is very simple and contains only one parameter. Here, we will also highlight the interesting fact that this approximation formula may be viewed as a special case of a modifier operator in the continuous-valued logic. The flexibility of the novel probability distribution function lays the foundations for its applications in different areas of science and in a wide range of modeling problems. We will point out that the pliant probability distribution function, as an alternative to the Weibull- and exponential distribution functions, can be used to model constant, monotonic and bathtub-shaped hazard functions in reliability theory. We will also demonstrate that a function transformed from the new probability distribution function can be utilized in the so-called kappa regression analysis method, which may be viewed as an alternative to logistic regression.

The remaining part of the paper is organized as follows. In Section 2, the omega function and the pliant probability distribution function are introduced and some important connections between the omega and the exponential functions are discussed. In Section 3, we demonstrate how the new probability distribution function can be utilized to approximate the Weibull, exponential, logistic and standard normal distribution functions and highlight some practical applications of our theoretical findings. Lastly, we will draw some key conclusions about the new probability distribution and make some suggestions for future research.

\section{The Pliant Probability Distribution Function}

Now, we will introduce a new four-parameter probability distribution function which we call the pliant probability distribution function. This novel function has the parameters $\alpha, \beta, \gamma$ and $d$, where $\alpha>0, d>0, \gamma \in\{-1,1\}$ and $\beta \in \mathbf{B}_{\gamma}$. First of all, we will define the domain $\mathbf{B}_{\gamma}$ of parameter $\beta$.

Definition 1. The set $\mathbf{B}_{\gamma}$ is given by

$\mathbf{B}_{\gamma}=\left\{b^{\frac{1}{2}(\gamma+1)}: b \in \mathbb{R}^{+}, \gamma \in\{-1,1\}\right\}$.

Notice that if $\gamma=1$, then $\mathbf{B}_{\gamma}=\mathbb{R}^{+}$, and if $\gamma=-1$, then $\mathbf{B}_{\gamma}=\{1\}$. From here on, a probability distribution function is always a cumulative distribution function (CDF). 
The pliant probability distribution function is founded on an auxiliary function that we call the omega function, the appropriate linear transformation of which is the generator function of certain unary operators in continuous-valued logic [7]. Firstly, we will introduce the omega function.

Definition 2. The omega function $\omega_{d}^{(\alpha, \beta)}(x)$ is given by

$\omega_{d}^{(\alpha, \beta)}(x)=\left(\frac{d^{\beta}+x^{\beta}}{d^{\beta}-x^{\beta}}\right)^{\frac{\alpha d^{\beta}}{2}}$,

where $\alpha, d \in \mathbb{R}, d>0, \beta \in \mathbf{B}_{\gamma}, x \in\left(\frac{d}{2}(\gamma-1), d\right), \gamma \in\{-1,1\}$.

Later, we will explain why this formula is so useful. Making use of this function, we can define the pliant probability distribution function.

Definition 3. The pliant probability distribution function $F_{P}(x ; \alpha, \beta, \gamma, d)$ is given by

$F_{P}(x ; \alpha, \beta, \gamma, d)= \begin{cases}0, & \text { if } x \leq \frac{d}{2}(\gamma-1) \\ \left(1-\gamma \omega_{d}^{(-\alpha, \beta)}(x)\right)^{\gamma}, & \text { if } x \in\left(\frac{d}{2}(\gamma-1), d\right) \\ 1, & \text { if } x \geq d,\end{cases}$

where $\alpha, d \in \mathbb{R}, \alpha>0, d>0, \beta \in \mathbf{B}_{\gamma}, \gamma \in\{-1,1\}$.

In order to demonstrate that the function $F_{P}(x ; \alpha, \beta, \gamma, d)$ is in fact a probability distribution function of a continuous random variable, we will describe the main properties of the omega function.

\subsection{Main Properties of the Omega Function}

Here, we state the most important properties of the omega function, namely domain, differentiability, monotonity, limits and convexity. Note that we demonstrated these properties in [11].

Domain We will utilize the omega function either with the domain $x \in(0, d)$, or with the domain $x \in(-d, d)$. Note that the domain $\mathbf{B}_{\gamma}$ of parameter $\beta$ is connected with the domain of $x$; that is, since $x \in\left(\frac{d}{2}(\gamma-1), d\right), \beta \in \mathbf{B}_{\gamma}$ and $\gamma \in\{-1,1\}$, one of the following two cases holds:

- if $\gamma=1$, then $x \in(0, d)$ and $\beta>0$

- if $\gamma=-1$, then $x \in(-d, d)$ and $\beta=1$.

Notice that we allow the parameter $\beta$ to have the value of 1 when $x \in(0, d)$, but $\beta$ just has the value of 1 when $x \in(-d, d)$.

Differentiability $\omega_{d}^{(\alpha, \beta)}(x)$ is differentiable in the interval $\left(\frac{d}{2}(\gamma-1), d\right)$. 
Monotonicity If $\alpha>0$, then $\omega_{d}^{(\alpha, \beta)}(x)$ is strictly monotonously increasing; if $\alpha<0$, then $\omega_{d}^{(\alpha, \beta)}(x)$ is strictly monotonously decreasing; if $\alpha=0$, then $\omega_{d}^{(\alpha, \beta)}(x)$ has a constant value of 1 in the interval $\left(\frac{d}{2}(\gamma-1), d\right)$.

\section{Limits}

- If $x \in(0, d)$ and $\beta>0$, then

$$
\begin{aligned}
& \lim _{x \rightarrow 0^{+}} \omega_{d}^{(\alpha, \beta)}(x)=1, \\
& \lim _{x \rightarrow d^{-}} \omega_{d}^{(\alpha, \beta)}(x)= \begin{cases}\infty, & \text { if } \alpha>0 \\
0, & \text { if } \alpha<0 .\end{cases}
\end{aligned}
$$

- If $x \in(-d, d)$ and $\beta=1$, then

$$
\begin{aligned}
& \lim _{x \rightarrow-d^{+}} \omega_{d}^{(\alpha, \beta)}(x)= \begin{cases}0, & \text { if } \alpha>0 \\
\infty, & \text { if } \alpha<0,\end{cases} \\
& \lim _{x \rightarrow d^{-}} \omega_{d}^{(\alpha, \beta)}(x)= \begin{cases}\infty, & \text { if } \alpha>0 \\
0, & \text { if } \alpha<0 .\end{cases}
\end{aligned}
$$

Convexity It can be shown that if $x \in(0, d)$ and $\beta>0$, then the convexity of the function $\omega_{d}^{(\alpha, \beta)}(x)$ in the interval $(0, d)$ is as follows:

- If $d^{2 \beta}<\frac{4\left(\beta^{2}-1\right)}{\alpha^{2} \beta^{2}}, \alpha \neq 0$, then $\omega_{d}^{(\alpha, \beta)}(x)$ is convex when $\alpha>0$ and $\omega_{d}^{(\alpha, \beta)}(x)$ is concave when $\alpha<0$.

- If $d^{2 \beta} \geq \frac{4\left(\beta^{2}-1\right)}{\alpha^{2} \beta^{2}}, \alpha \neq 0$, then we can distinguish the following cases:

- if $\alpha>0$ and $0<\beta<1$, then $\omega_{d}^{(\alpha, \beta)}(x)$ changes its shape from concave to convex at $x_{r}$

- if $\alpha>0$ and $\beta \geq 1$, then $\omega_{d}^{(\alpha, \beta)}(x)$ is convex

- if $\alpha<0,0<\beta \leq 1$ and $x_{r}<d$, then $\omega_{d}^{(\alpha, \beta)}(x)$ changes its shape from convex to concave at $x_{r}$

- if $\alpha<0,0<\beta \leq 1$ and $x_{r} \geq d$, then $\omega_{d}^{(\alpha, \beta)}(x)$ is convex

- if $\alpha<0, \beta>1$ and $x_{r}<d$, then $\omega_{d}^{(\alpha, \beta)}(x)$ changes its shape from concave to convex at $x_{l}$ and from convex to concave at $x_{r}$

- if $\alpha<0, \beta>1$ and $x_{r} \geq d$, then $\omega_{d}^{(\alpha, \beta)}(x)$ changes its shape from concave to convex at $x_{l}$, 
where

$$
\begin{aligned}
& x_{l}=\left(\frac{-\alpha \beta d^{2 \beta}-\sqrt{\alpha^{2} \beta^{2} d^{4 \beta}-4\left(\beta^{2}-1\right) d^{2 \beta}}}{2(\beta+1)}\right)^{1 / \beta} \\
& x_{r}=\left(\frac{-\alpha \beta d^{2 \beta}+\sqrt{\alpha^{2} \beta^{2} d^{4 \beta}-4\left(\beta^{2}-1\right) d^{2 \beta}}}{2(\beta+1)}\right)^{1 / \beta} .
\end{aligned}
$$

It can be also shown that if $x \in(-d, d)$ and $\beta=1$, then the shape of the function $\omega_{d}^{(\alpha, \beta)}(x)$ in the interval $(-d, d)$ is as follows:

- if $|\alpha| d \geq 2$, then $\omega_{d}^{(\alpha, \beta)}(x)$ is convex

- if $\alpha>0$ and $\alpha d<2$, then $\omega_{d}^{(\alpha, \beta)}(x)$ changes from concave to convex at $-\alpha d^{2} / 2$

- if $\alpha<0$ and $\alpha d>-2$, then $\omega_{d}^{(\alpha, \beta)}(x)$ changes from convex to concave at $-\alpha d^{2} / 2$.

Based on the above-mentioned properties of the omega function, the basic semantics of the parameters $\alpha, \beta$ and $d$ can be summarized as follows. The parameter $d$ determines the domain of the omega function (either $x \in(-d, d)$, or $x \in(0, d)$ ), the parameter $\alpha$ influences its monotonicity and steepness, while the parameter $\beta$ also affects the steepness of $\omega_{d}^{(\alpha, \beta)}(x),(\alpha, d \in \mathbb{R}, d>0, \beta>0)$. Figure 1 shows plots of some omega functions.
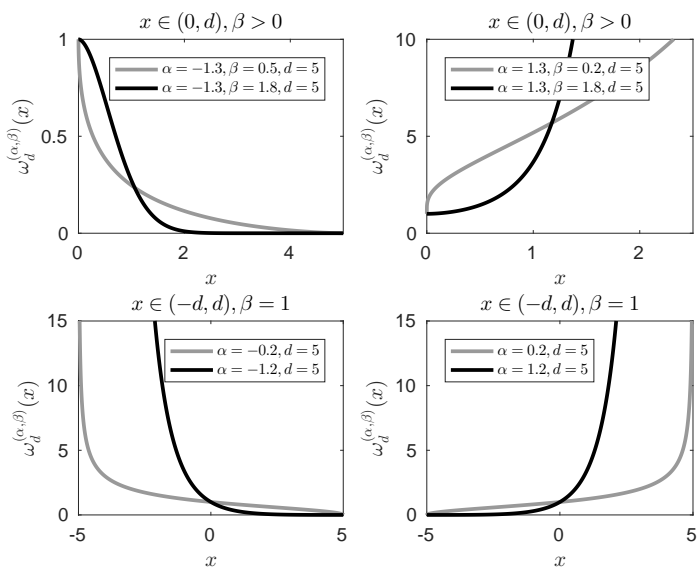

Figure 1

Plots of some omega functions 


\subsubsection{The Generalized Exponential Differential Equation}

Next, we will introduce the generalized exponential differential equation and show how it is related to the exponential function $f(x)=\exp \left(\alpha x^{\beta}\right),(\alpha, \beta \in \mathbb{R}, \beta>0)$ and to the omega function.

Definition 4. We define the generalized exponential differential equation as

$\frac{\mathrm{d} f(x)}{\mathrm{d} x}=\alpha \beta x^{\beta-1}\left(\frac{d^{2 \beta}}{d^{2 \beta}-x^{2 \beta}}\right)^{\varepsilon} f(x)$,

where $\alpha, d \in \mathbb{R}, d>0, \beta \in \mathbf{B}_{\gamma}, x \in\left(\frac{d}{2}(\gamma-1), d\right), \gamma \in\{-1,1\}, \varepsilon \in\{0,1\}, f(x)>0$.

Lemma 1 (Lemma 2 in [11]). The solutions of the generalized exponential differential equation are:

$f(x)= \begin{cases}C \exp \left(\alpha x^{\beta}\right), & \text { if } \varepsilon=0 \\ C\left(\frac{d^{\beta}+x^{\beta}}{d^{\beta}-x^{\beta}}\right)^{\frac{a d}{2}}, & \text { if } \varepsilon=1,\end{cases}$

where $C \in \mathbb{R}$ and $C>0$.

Proof. See the proof of Lemma 2 in [11].

\subsubsection{Connections Between the Exponential and Omega Functions}

Lemma 1 suggests that there is a key connection between the exponential function $f(x)=\exp \left(\alpha x^{\beta}\right)$ and the omega function. Namely, the solution of the generalized exponential differential equation with $\varepsilon=0$ and $C=1$ is simply the exponential function $f(x)=\exp \left(\alpha x^{\beta}\right)$, while the solution of (10) with $\varepsilon=1, C=1$ is the omega function. Furthermore, if $d$ is much greater than $x$, then

$\frac{d^{2 \beta}}{d^{2 \beta}-x^{2 \beta}} \approx 1$

In this case the generalized exponential differential equation for $\varepsilon=1$ becomes the following approximate equation:

$\frac{\mathrm{d} f(x)}{\mathrm{d} x} \approx \alpha \beta x^{\beta-1} f(x)$,

which is nearly the generalized exponential differential equation with $\varepsilon=0$, the solution of which is the exponential function $f(x)=\exp \left(\alpha x^{\beta}\right)$. The following proposition provides the theoretical basis for this result (see Theorem 1 in [11]).

Proposition 1 (Theorem 1 in [11]). For any $x \in\left(\frac{d}{2}(\gamma-1), d\right)$,

$\lim _{d \rightarrow \infty} \omega_{d}^{(\alpha, \beta)}(x)=\exp \left(\alpha x^{\beta}\right)$,

where $\alpha, d \in \mathbb{R}, d>0, \beta \in \mathbf{B}_{\gamma}, \gamma \in\{-1,1\}$. 
Proof. See the proof of Theorem 1 in [11].

Based on Proposition 1, we can state that the asymptotic omega function is just the exponential function $f(x)=\exp \left(\alpha x^{\beta}\right)$. Actually, if $x \ll d$, then $\omega_{d}^{(\alpha, \beta)}(x) \approx$ $\exp \left(\alpha x^{\beta}\right)$; that is, if $d$ is sufficiently large, then the omega function suitably approximates the exponential function $f(x)=\exp \left(\alpha x^{\beta}\right)$.

\section{Approximations and their Applications}

Here, we will demonstrate how the pliant probability distribution function $F_{P}(x ; \alpha, \beta, \gamma, d)$ can be utilized for approximating the Weibull, exponential, logistic and standard normal probability distribution functions. First, we will show that the function $F_{P}(x ; \alpha, \beta, \gamma, d)$ is in fact a probability distribution function.

Lemma 2. The function $F_{P}(x ; \alpha, \beta, \gamma, d)$ given by Definition 3 is a probability distribution function.

Proof. The function $F_{P}(x ; \alpha, \beta, \gamma, d)$ is a probability distribution function if it is:

1. monotonously increasing

2. left continuous,

3. and $\lim _{x \rightarrow-\infty} F_{P}(x ; \alpha, \beta, \gamma, d)=0$ and $\lim _{x \rightarrow+\infty} F_{P}(x ; \alpha, \beta, \gamma, d)=1$.

These properties of the function $F_{P}(x ; \alpha, \beta, \gamma, d)$ readily follow from properties of the omega function $\omega_{d}^{(\alpha, \beta)}(x)$.

We have demonstrated that the omega function in the asymptotic limit is just the exponential function $f(x)=\exp \left(\alpha x^{\beta}\right)$, where $\beta>0$. Exploiting this result and the fact the function $F_{P}(x ; \alpha, \beta, \gamma, d)$ is a probability distribution function, we will show how the pliant probability distribution function can be used to approximate some well-known probability distribution functions that include exponential terms. From now on, we will use the notation $\xi \sim D_{p}(\alpha, \beta, \gamma, d)$ to indicate that the random variable $\xi$ has a pliant probability distribution with the parameters $\alpha, \beta, \gamma, d$. That is, if $\xi \sim D_{p}(\alpha, \beta, \gamma, d)$, then $P(\xi<x)=F_{P}(x ; \alpha, \beta, \gamma, d)$ for any $x \in \mathbb{R}$.

\subsection{Approximation to the Weibull probability Distribution Function}

The 2-parameter distribution function $F_{W}(x ; \beta, \lambda)$ of the random variable which has a Weibull probability distribution is usually given by the formula:

$F_{W}(x ; \beta, \lambda)= \begin{cases}0, & \text { if } x \leq 0 \\ 1-\exp \left(-(x / \lambda)^{\beta}\right) & \text { if } x>0\end{cases}$ 
where $\beta, \lambda \in \mathbb{R}$ and $\beta, \lambda>0$ are the shape and scale parameters of the distribution, respectively $[24,33]$. Making the substitution $\alpha=\lambda^{-\beta},(15)$ may be written in the form

$F_{W}(x ; \alpha, \beta)= \begin{cases}0, & \text { if } x \leq 0 \\ 1-\exp \left(-\alpha x^{\beta}\right), & \text { if } x>0,\end{cases}$

where $\alpha, \beta \in \mathbb{R}, \alpha, \beta>0$. Hereafter, we will use this alternative definition of the 2parameter distribution function of the random variable that has a Weibull probability distribution. Furthermore, we will use the notation $\eta \sim W(\alpha, \beta)$ to indicate that the random variable $\eta$ has a Weibull probability distribution with the parameters $\alpha, \beta>0$; that is, if $\eta \sim W(\alpha, \beta)$, then $P(\eta<x)=F_{W}(x ; \alpha, \beta)$ for any $x \in \mathbb{R}$.

Proposition 2. If $\xi \sim D_{p}(\alpha, \beta, \gamma, d), \eta \sim W(\alpha, \beta)$ and $\gamma=1$, then for any $x \in \mathbb{R}$,

$\lim _{d \rightarrow \infty} P(\xi<x)=P(\eta<x)$,

where $\alpha, d \in \mathbb{R}, \alpha, d>0, \beta \in \mathbf{B}_{\gamma}$.

Proof. Utilizing the definitions of $F_{P}(x ; \alpha, \beta, \gamma, d)$ and $\mathbf{B}_{\gamma}$, if $\gamma=1$, then the pliant probability distribution function $F_{P}(x ; \alpha, \beta, \gamma, d)$ may be written as

$F_{P}(x ; \alpha, \beta, \gamma, d)= \begin{cases}0, & \text { if } x \leq 0 \\ 1-\omega_{d}^{(-\alpha, \beta)}(x), & \text { if } x \in(0, d) \\ 1, & \text { if } x \geq d .\end{cases}$

Let $x \in \mathbb{R}$ be fixed. We will now distinguish the following two cases.

1. If $x \leq 0$, then $F_{P}(x ; \alpha, \beta, \gamma, d)=F_{W}(x ; \beta, \lambda)$ holds by definition.

2. If $x \in(0, d), d>0$, then

$$
F_{P}(x ; \alpha, \beta, \gamma, d)=1-\omega_{d}^{(-\alpha, \beta)}(x) .
$$

Next, following Proposition 1 , if $d \rightarrow \infty$, then

$$
F_{P}(x ; \alpha, \beta, \gamma, d)=1-\omega_{d}^{(-\alpha, \beta)}(x) \rightarrow 1-\exp \left(-\alpha x^{\beta}\right)=F_{W}(x ; \alpha, \beta) .
$$

That is,

$$
\lim _{d \rightarrow \infty} P(\xi<x)=P(\eta<x) .
$$

Some example plots of Weibull probability distribution functions and their approximations by pliant probability distribution functions are shown in Figure 2. In each subplot of Figure 2, the left hand side scale is associated with functions $F_{W}(x ; \alpha, \beta)$ and $F_{P}(x ; \alpha, \beta, \gamma, d)$, while the right hand side scale is related to the difference function $F_{W}(x ; \alpha, \beta)-F_{P}(x ; \alpha, \beta, \gamma, d)$. We can see that, in line with Proposition 2 , the goodness of approximation improves with the increasing value of the parameter $d$. 


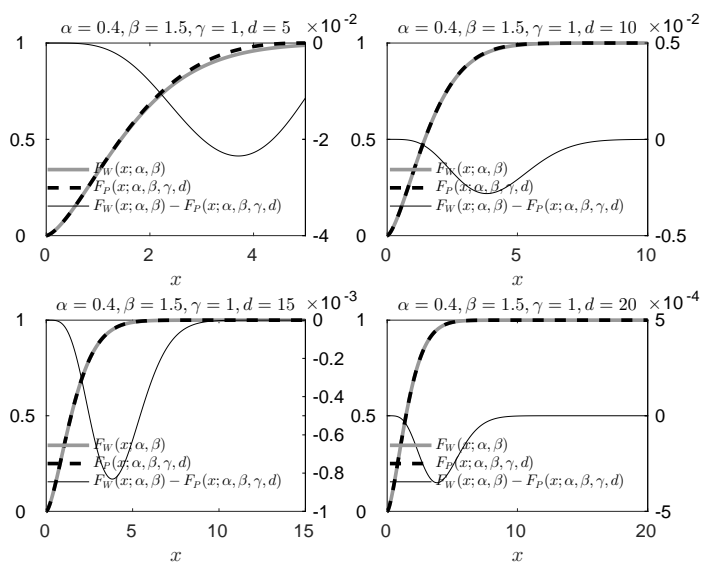

Figure 2

Examples of Weibull- and pliant probability distribution function plots

\subsubsection{Applications in Reliability Theory}

Let the continuous random variable $\tau$ be the time-to-first-failure of a component or system. In reliability theory, the failure rate function $h(t)$ for $\tau$ is given by

$h(t)=\lim _{\Delta t \rightarrow 0} \frac{F(t+\Delta t)-F(t)}{\Delta t R(t)}=\frac{f(t)}{R(t)}$,

where $f(t)$ is the probability density function of $\tau$. The hazard function $h(t)$ is also called the failure rate function. In practice, the quantity $h(t) \Delta t$ represents the conditional probability that a component or a system will fail in the time interval $(t, t+\Delta t]$, given that it has survived up to time $t,(t, \Delta t>0)$.

A typical hazard function curve of a component or a system is bathtub-shaped; that is, it can be divided into three distinct phases called the infant mortality period, useful life, and wear-out period. It is typical that the probability distribution of $\tau$ is different in the three characteristic phases of the bathtub-shaped hazard function. If $\tau$ has a Weibull probability distribution with the parameters $\alpha, \beta>0$, then using (22), the hazard function $h_{W}(t ; \alpha, \beta)$ of $\tau$ (which we call the Weibull hazard function) is

$h_{W}(t ; \alpha, \beta)=\frac{f_{W}(t ; \alpha, \beta)}{1-F_{W}(t ; \alpha, \beta)}=\frac{\alpha \beta t^{\beta-1} \exp \left(-\alpha t^{\beta}\right)}{\exp \left(-\alpha t^{\beta}\right)}=\alpha \beta t^{\beta-1}$,

where $f_{W}(t ; \alpha, \beta)$ is the probability density function of $\tau$. Equation (23) suggests an important property of the hazard function $h(t ; \alpha, \beta)$. Namely,

- if $0<\beta<1$, then $h_{W}(t ; \alpha, \beta)$ is decreasing with respect to time

- if $\beta=1$, then $h_{W}(t ; \alpha, \beta)$ is constant and has a value of $\alpha$

- if $\beta>1$, then $h_{W}(t ; \alpha, \beta)$ is increasing with respect to time. 
That is, if $\tau \sim W(\alpha, \beta)$, then the failure rate function $h_{W}(t ; \alpha, \beta)$, with appropriate values of its parameters, can characterize each of the three phases of a bathtubshaped failure rate curve quite well. On the one hand, this flexibility of the Weibull probability distribution makes it suitable for modeling the probability distribution of time-to-first-failure random variable in a wide range of reliability analyses [23]. On the other hand, the Weibull hazard function $h_{W}(t ; \alpha, \beta)$ is either monotonic or constant; that is, its curve cannot be bathtub-shaped. However, lifetime data of a component or system typically require non-monotonic shapes like the bathtub shape. Many modifications have been suggested for the Weibull probability distribution in order to have non-monotonic shapes. See, for example, the publications [17], [25], [22], [34], [37], [14], [13], [18], [27], [28], [4], [6]. A comprehensive review of the known modifications to the Weibull probability distribution can be found in a quite recent article [1]. Here, we will demonstrate that the hazard function of the pliant probability distribution can be used to model both monotonic and bathtub-shaped hazard rate curves.

Now let us assume that $\tau$ has a pliant probability distribution with the parameters $\alpha, d>0, \gamma=1$. In this case, $\beta>0$ and the hazard function of $\tau$, which we will call the pliant hazard function, is

$$
\begin{gathered}
h_{P}(t ; \alpha, \beta, d)=\frac{f_{P}(t ; \alpha, \beta, d)}{1-F_{P}(t ; \alpha, \beta, d)}=\frac{\alpha \beta t^{\beta-1} \frac{d^{2 \beta}}{d^{2 \beta}-t^{2 \beta}} \omega_{d}^{(-\alpha, \beta)}(t)}{\omega_{d}^{(-\alpha, \beta)}(t)}= \\
=\alpha \beta t^{\beta-1} \frac{d^{2 \beta}}{d^{2 \beta}-t^{2 \beta}},
\end{gathered}
$$

if $0<t<d$, where $f_{P}(t ; \alpha, \beta, d)$ is the probability density function of $\tau$. Utilizing (23) and (24), the pliant hazard function $h_{P}(t ; \alpha, \beta, d)$ may be written as

$h_{P}(t ; \alpha, \beta, d)=h_{W}(\alpha, \beta) g(t ; \beta, d)$,

where

$g(t ; \beta, d)=\frac{d^{2 \beta}}{d^{2 \beta}-t^{2 \beta}}$,

and $\alpha, \beta, d>0, t \in(0, d)$. That is, the pliant hazard function may be interpreted as the Weibull hazard function multiplied by the corrector function $g(t ; \beta, d)$. The pliant hazard function $h_{P}(t ; \alpha, \beta, d)$ has some key properties that make it suitable for modeling bathtub-shaped failure rate curves. The following lemma allows us to utilize the pliant hazard function as an alternative to the Weibull hazard function.

Lemma 3. For any $t \in(0, d)$, if $d \rightarrow \infty$, then $h_{P}(t ; \alpha, \beta, d) \rightarrow h_{W}(t ; \alpha, \beta)$, where $\alpha, \beta, d>0$.

Proof. If $t \in(0, d)$ is fixed and $d \rightarrow \infty$, then $g(t ; \beta, d) \rightarrow 1$ and so

$h_{P}(t ; \alpha, \beta, d)=h_{W}(\alpha, \beta) g(t ; \beta, d) \rightarrow h_{W}(\alpha, \beta)$. 
The practical implication of this result is as follows. Since the Weibull hazard function can be utilized as a model for each phase of a bathtub-shaped failure rate curve and $h_{P}(t ; \alpha, \beta, d) \approx h_{W}(\alpha, \beta)$, if $t$ is small compared to $d$, the pliant hazard function can also model each phase of the same bathtub-shaped failure rate curve, if $d$ is sufficiently large. That is, the pliant hazard function, as an alternative to the Weibull hazard function, can be utilized as a phase-by-phase model of a bathtub-shaped failure rate curve.

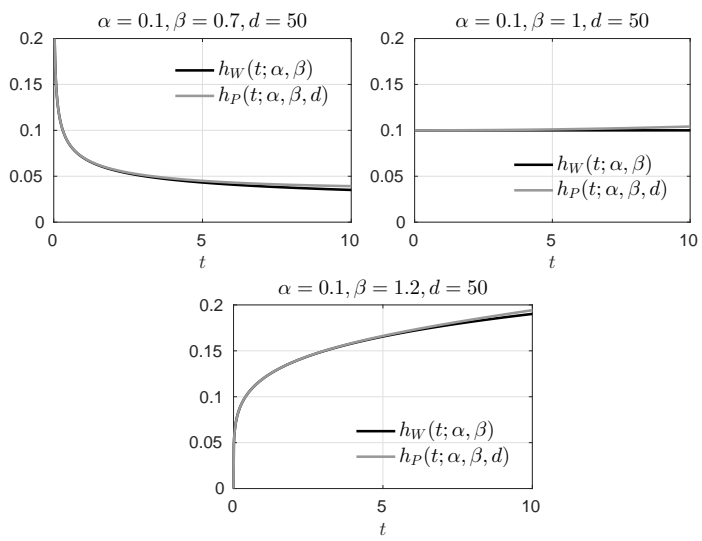

Figure 3

Plots of Weibull- and pliant hazard functions (Figure 5 in [11])

Figure 3 shows how the Weibull- and pliant hazard function curves can model each characteristic phase of a failure rate curve. The plots in Figure 3 demonstrate the results of the previous lemma; that is, if $t \ll d$, then the pliant hazard function approximates the Weibull hazard function quite well.

The next lemma shows how that the pliant hazard function $h_{P}(t ; \alpha, \beta, d)$ can be utilized as a model for all the three phases of a bathtub-shaped failure rate curve.

Lemma 4 (Lemma 5 in [11]). If $0<\beta<1$, then $h_{P}(t ; \alpha, \beta, d)$ is strictly convex in the interval $(0, d)$ and $h_{P}(t ; \alpha, \beta, d)$ has its minimum at

$t_{0}=d\left(\frac{1-\beta}{1+\beta}\right)^{\frac{1}{2 \beta}}$.

Proof. The lemma follows from the elementary properties of the pliant hazard function $h_{P}(t ; \alpha, \beta, d)$ by using its first and second derivatives.

Figure 4 shows some plots of the pliant hazard function $h_{P}(t ; \alpha, \beta, d)$ with $0<\beta<$ 1.

Based on the above properties of the pliant hazard function, we may conclude that the pliant probability distribution with $\gamma=1$ can be employed to describe the probability distribution of the time-to-first-failure random variable in each characteristic phase of a bathtub-shaped failure rate curve. Moreover, we have two possibilities for 


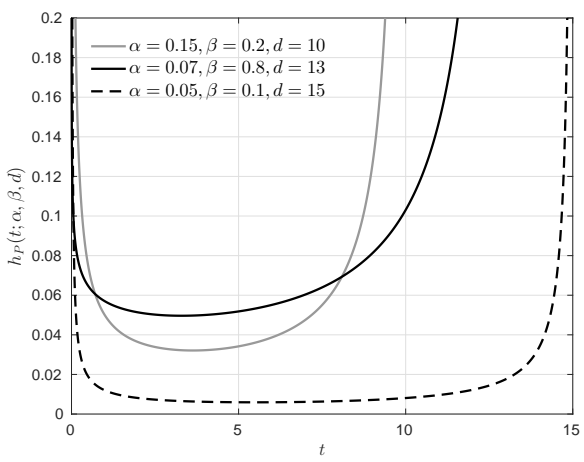

Figure 4

Examples of bathtub-shaped pliant hazard function plots (Figure 6 in [11])

modeling a bathtub-shaped failure rate curve. Namely, either we piecewise describe each phase by a pliant hazard function, or we apply one pliant hazard function that models the entire failure rate curve. It is worth noting here that the Weibull distribution is widely applied to model reliability of software systems (see, e.g. [19, 29]), therefore, our pliant probability distribution, as an alternative to the Weibull probability distribution, can also be applied in this area.

\subsection{Approximation to the Exponential probability Distribution Function}

If the random variable $\eta$ has an exponential probability distribution with the parameter $\alpha>0$, then the probability distribution function $F_{\text {exp }}(x ; \alpha)$ of $\eta$ is given by

$F_{\text {exp }}(x ; \alpha)= \begin{cases}0, & \text { if } x \leq 0 \\ 1-\exp (-\alpha x), & \text { if } x>0\end{cases}$

[24]. We will use the notation $\eta \sim \exp (\alpha)$ to indicate that the random variable $\eta$ has an exponential probability distribution with the parameter $\alpha>0$. The probability distribution function $F_{\text {exp }}(x ; \alpha)$ is a special case of the Weibull probability distribution function $F_{W}(x ; \alpha, \beta)$. Namely, if $\beta=1$, then $F_{\text {exp }}(x ; \alpha)=F_{W}(x ; \alpha, \beta)$. Based on this, we can state the following proposition.

Proposition 3. If $\xi \sim D_{p}(\alpha, \beta, \gamma, d), \eta \sim \exp (\alpha), \gamma=1$ and $\beta=1$, then for any $x \in \mathbb{R}$,

$\lim _{d \rightarrow \infty} P(\xi<x)=P(\eta<x)$,

where $\alpha, d \in \mathbb{R}, \alpha, d>0$.

Proof. The proposition follows from Proposition 2. 
This result tells us that if $\gamma=1, \beta=1$, then the asymptotic pliant probability distribution function, for the parameter $d$, is just the exponential probability distribution function. Note that the pliant probability distribution is a generalization of the epsilon probability distribution that we introduced in [10].

\subsubsection{Applications in Reliability Theory}

The exponential probability distribution plays a significant role in the theory and practice of reliability management $[36,32]$. This distribution also appears frequently in lifetime and reaction time studies. Here, we will discuss how the pliant probability distribution, as an alternative to the exponential distribution, can be applied to model failure rates.

Since the exponential probability distribution may be viewed as a special case of the Weibull probability distribution, namely when $\beta=1$, utilizing the Weibull hazard function in (23) with $\beta=1$ gives us the hazard function $h_{\exp }(t ; \alpha)$ of the exponential distribution with the parameter $\alpha>0$ :

$h_{\text {exp }}(t ; \alpha)=h_{W}(t ; \alpha, \beta)_{\mid \beta=1}=\alpha \beta t_{\mid \beta=1}^{\beta-1}=\alpha$.

That is, if $\tau \sim \exp (\alpha)$, then the hazard function $h_{\exp }(t ; \alpha)$ of $\tau$ is constant with the value $\alpha$. Based on Lemma 3, the pliant hazard function tends to the Weibull hazard function, if $d \rightarrow \infty$. Applying this results for the special case where $\beta=1$, we get the following reduced pliant hazard function

$h_{P}(t ; \alpha)=h_{P}(t ; \alpha, \beta)_{\mid \beta=1}=\left.\alpha \beta t^{\beta-1} \frac{d^{2 \beta}}{d^{2 \beta}-t^{2 \beta}}\right|_{\beta=1}=\alpha \frac{d^{2}}{d^{2}-t^{2}}$,

where $t \in(0, d)$. There are two key properties of the pliant hazard function $h_{P}(t ; \alpha)$ that should be mentioned here. These are:

1. If $t \in(0, d)$ is fixed and $d \rightarrow \infty$, then $h_{P}(t ; \alpha) \rightarrow \alpha$.

2. $h_{P}(t ; \alpha)$ is monotonously increasing in the interval $(0, d)$.

The practical implications of the above properties of the hazard function $h_{P}(t ; \alpha)$ are as follows. If $d$ is sufficiently large compared to $t$, then $h_{P}(t ; \alpha)$ is approximately constant with the value $\alpha$; that is, function $h_{P}(t ; \alpha)$ can be utilized to model the quasi constant second phase of a bathtub-shaped failure rate curve. As $h_{P}(t ; \alpha)$ is monotonously increasing in the interval $(0, d)$, it can also be treated as a model of the increasing third phase of a bathtub-shaped hazard curve (see also [10]).

\subsection{Approximation to the Logistic Probability Distribution Function}

The 2-parameter probability distribution function $F_{L}(x ; \mu, s)$ of the random variable that has a logistic probability distribution is commonly given by

$F_{L}(x ; \mu, s)=\frac{1}{1+\exp \left(-\frac{x-\mu}{s}\right)}$, 
where $\mu, s \in \mathbb{R}$ and $s>0$ are the location and scale parameters of the distribution, respectively $[16,3]$.

By applying the $\alpha=1 / s$ substitution and setting the location parameter $\mu$ to zero, (33) may be written as

$F_{L}(x ; \alpha)=\frac{1}{1+\exp (-\alpha x)}$

where $\alpha \in \mathbb{R}, \alpha>0$. From now on, we will utilize the logistic probability distribution function in the latter form and use the notation $\eta \sim L(\alpha)$ to indicate that the random variable $\eta$ has a logistic distribution with the parameter $\alpha>0$; that is, $P(\eta<x)=F_{L}(x ; \alpha)$. The following proposition will demonstrate that the pliant probability distribution function can be used to approximate the logistic distribution function $F_{L}(x ; \alpha)$.

Proposition 4. If $\xi \sim D_{p}(\alpha, \beta, \gamma, d), \eta \sim L(\alpha), \gamma=-1$, then for any $x \in \mathbb{R}$,

$\lim _{d \rightarrow \infty} P(\xi<x)=P(\eta<x)$,

where $\alpha, d \in \mathbb{R}, \alpha, d>0$.

Proof. Since $\gamma=-1$ and $\beta=1$, the pliant probability distribution function $F_{P}(x ; \alpha, \beta, \gamma, d)$ may be written as

$F_{P}(x ; \alpha, \beta, \gamma, d)= \begin{cases}0, & \text { if } x \leq 0 \\ \frac{1}{1+\omega_{d}^{(-\alpha, \beta)}(x)}, & \text { if } x \in(-d, d) \\ 1, & \text { if } x \geq d .\end{cases}$

Let $x \in \mathbb{R}$ be fixed. Now, utilizing the fact that $\beta=1$ and exploiting Proposition 1 gives

$F_{P}(x ; \alpha, \beta, \gamma, d)=\frac{1}{1+\omega_{d}^{(-\alpha, \beta)}(x)} \stackrel{d \rightarrow \infty}{\longrightarrow} \frac{1}{1+\exp (-\alpha x)}=F_{L}(x ; \alpha)$.

This result means that

$\lim _{d \rightarrow \infty} P(\xi<x)=P(\eta<x)$.

Based on Proposition 4, we can state that the pliant probability distribution function $F_{P}(x ; \alpha, \beta, \gamma, d)$ can be used to approximate the logistic distribution function $F_{L}(x ; \alpha)$. A few sample plots of logistic probability distribution functions and their approximations by pliant probability distribution functions are shown in Figure 5. In each subplot of Figure 5, the left hand side scale is associated with functions $F_{L}(x ; \alpha)$ and $F_{P}(x ; \alpha, \beta, \gamma, d)$, while the right hand side scale is related to the difference function $F_{L}(x ; \alpha)-F_{P}(x ; \alpha, \beta, \gamma, d)$. The plots show how the goodness of approximation improves with increasing values of the parameter $d$. 


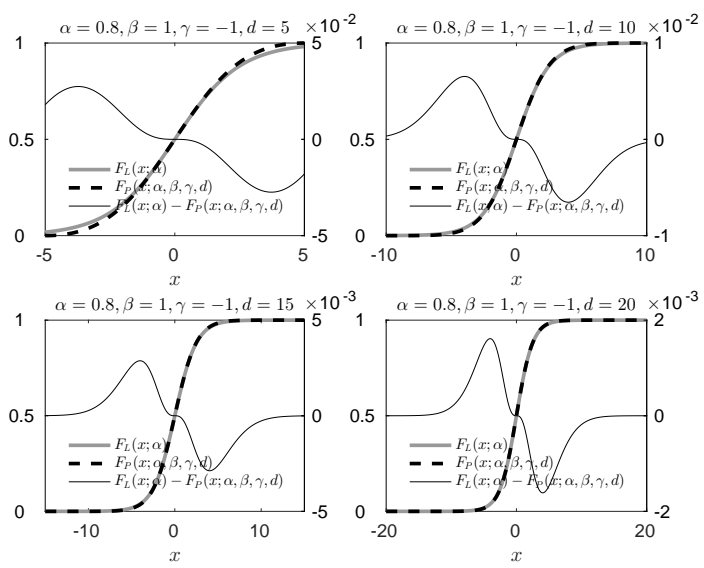

Figure 5

Examples of logistic- and pliant probability distribution function plots

\subsubsection{The Kappa Regression Function}

The logistic function as a regression function has a wide range of applications in many fields, including economics, business, biology, the medical sciences and engineering. Here, we will demonstrate that a function which can be derived from the pliant probability distribution function may be viewed as an alternative to the logistic regression function. If $\gamma=-1$, then $\beta=1$ and the formula of the pliant probability distribution function for $x \in(-d, d)$ gives the following $\kappa_{d}^{(\alpha)}(x)$ function:

$\kappa_{d}^{(\alpha)}(x)=\frac{1}{1+\left(\frac{d+x}{d-x}\right)^{\frac{-\alpha d}{2}}}$,

where $d>0$ and $\alpha \in \mathbb{R}$. Notice that here we allow $\alpha$ to take any value. Let $Y$ be a dichotomous random variable, and let 0 and 1 code its possible values. Here, we model the conditional probability $P(Y=k \mid x)$ as a function of the independent variable $x ; x \in(a, b), k \in\{0,1\}$. In our model, which we call the kappa regression model [9], the odds $O(x)$ are given by

$O(x)=\frac{P(Y=1 \mid x)}{1-P(Y=1 \mid x)}=C^{\prime}\left(\frac{x-a}{b-x}\right)^{\lambda}$,

where $\lambda \in \mathbb{R}$ and $C^{\prime}>0$. If $C^{\prime}$ is written in the form $C^{\prime}=\exp (-c)$, then from (40):

$P(Y=1 \mid x)=\frac{1}{1+\exp (c)\left(\frac{b-x}{x-a}\right)^{\lambda}}=\kappa(x ; \lambda, a, b, c)$.

Notice that the kappa function $\kappa_{d}^{(\alpha)}(y)$ can be derived from the kappa function $\kappa(x ; \lambda, a, b, c)$ by setting $c=0, \lambda=\alpha d / 2$ and applying the linear transformation $x=\frac{y+d}{2 d}(b-a)+a$ with $d>0$. 
In the logistic regression model, the odds $O(x)$ are modeled by

$O(x)=\frac{P(Y=1 \mid x)}{1-P(Y=1 \mid x)}=\exp \left(\beta_{1} x+\beta_{0}\right)$,

and so

$P(Y=1 \mid x)=\frac{1}{1+\exp \left(-\beta_{1} x-\beta_{0}\right)}$,

where $\beta_{0}, \beta_{1} \in \mathbb{R}$. The next lemma lays the foundations for using kappa regression as an alternative to logistic regression.

Lemma 5. If $a<b,(a+b) / 2=x_{0}$ is constant, $\lambda=\beta_{1} \frac{\left(x_{0}-a\right)\left(b-x_{0}\right)}{b-a}$ and $a \rightarrow-\infty$, $b \rightarrow+\infty$ so that $(a+b) / 2=x_{0}$, then for any $x \in(a, b)$,

$C^{\prime}\left(\frac{x-a}{b-x}\right)^{\lambda} \rightarrow \exp \left(\beta_{1} x+\beta_{0}\right)$,

where $\beta_{0}=\ln C^{\prime}-\beta_{1} x_{0},\left(C^{\prime}>0\right)$.

Proof. Here, $a \rightarrow-\infty, b \rightarrow+\infty$ so that $(a+b) / 2=x_{0}$; that is, $a \rightarrow-\infty$ and $b \rightarrow+\infty$ may be written as $a=x_{0}-\Delta, b=x_{0}+\Delta$, where $\Delta \rightarrow \infty$. Then, if the conditions of the lemma are satisfied,

$$
\begin{gathered}
\lim _{\substack{a \rightarrow-\infty \\
b \rightarrow+\infty}}\left(C^{\prime}\left(\frac{x-a}{b-x}\right)^{\lambda}\right)=\exp \left(\beta_{1} x_{0}+\beta_{0}\right) \lim _{\substack{a \rightarrow-\infty \\
b \rightarrow+\infty}}\left(\frac{x-a}{b-x}\right)^{\beta_{1} \frac{\left(x_{0}-a\right)\left(b-x_{0}\right)}{b-a}}= \\
=\exp \left(\beta_{1} x_{0}+\beta_{0}\right) \lim _{\Delta \rightarrow \infty}\left(\frac{x-x_{0}+\Delta}{\Delta-\left(x-x_{0}\right)}\right)^{\beta_{1} \frac{\Delta}{2}} .
\end{gathered}
$$

Similar to the proof of Proposition 1, it can be shown that

$$
\lim _{\Delta \rightarrow \infty}\left(\frac{x-x_{0}+\Delta}{\Delta-\left(x-x_{0}\right)}\right)^{\beta_{1} \frac{\Delta}{2}}=\exp \left(\beta_{1}\left(x-x_{0}\right)\right),
$$

and so the chain in (45) can be continued as

$$
\begin{gathered}
\exp \left(\beta_{1} x_{0}+\beta_{0}\right) \lim _{\Delta \rightarrow \infty}\left(\frac{x-x_{0}+\Delta}{\Delta-\left(x-x_{0}\right)}\right)^{\beta_{1} \frac{\Delta}{2}}= \\
=\exp \left(\beta_{1} x_{0}+\beta_{0}\right) \exp \left(\beta_{1}\left(x-x_{0}\right)\right)=\exp \left(\beta_{1} x+\beta_{0}\right) .
\end{gathered}
$$

Hence, we have demonstrated that logistic regression may be regarded as asymptotic kappa regression. Based on our findings, kappa regression may be treated as a quasi logistic regression, where the explanatory variable is defined over a bounded subset of the real numbers. This property of kappa regression is advantageous in situations where the explanatory variable is defined over a bounded subset of real numbers, the empirical conditional probabilities exhibit an asymmetric trend and there are empirical conditional probabilities very close to zero or one at the terminal locations of the domain of the explanatory variable [9]. 


\subsection{Approximation to the Standard Normal Probability Distri- bution Function}

The probability distribution function $\Phi(x)$ of the standard normal random variable is given by

$\Phi(x)=\frac{1}{\sqrt{2 \pi}} \int_{-\infty}^{x} \exp \left(-\frac{t^{2}}{2}\right) \mathrm{d} t$.

We will use the common notation $\eta \sim N(0,1)$ to indicate that the random variable $\eta$ has the standard normal probability distribution. The fact that the probability distribution function $\Phi(x)$ cannot be expressed in a closed form and the practical needs for computing its values provided the motivation for researchers and practitioners to approximate the standard normal probability distribution function. These research efforts resulted in an extremely wide range of approximations with various applications. Most of the approximations belong to the group of ad-hoc methods which typically utilize an a priori selected parametric function and apply various mathematical techniques to estimate the parameters in order minimize the approximation error. [21], [30] and [35] gave comprehensive overviews of the approximation formulas in their reviews. In general, we can say that the accuracy of approximations increases with the complexity of formulas and with the number of parameters they have.

Among the many ad-hoc approximations available, the 1-parameter logistic function, which has the same form as the logistic probability distribution function $F_{L}(x ; \alpha)$, can also be applied to approximate the standard normal probability distribution function (see, for example, the books of [31], [3], and [15], and the paper of [5]). Here, we will utilize Tocher's approximation and the pliant probability distribution function to obtain a novel approximation (which has only one parameter and a very simple formula) to the standard normal probability distribution function. Tocher's approximation, which we denote by $\Phi_{T}(x)$, utilizes the logistic probability distribution function $F_{L}(x ; \alpha)$ with $\alpha=2 \sqrt{2 / \pi}$. That is,

$\Phi_{T}(x)=\frac{1}{1+\exp (-2 \sqrt{2 / \pi} x)}$.

It is worth mentioning here that the probability density function of Tocher's approximation can be derived from sigmoid fuzzy membership functions by using operators of continuous-valued logic [8]. Note that setting $\alpha$ to $2 \sqrt{2 / \pi}$ ensures that function $\Phi_{T}(x)$ is identical with function $\Phi(x)$ to first order at $x=0$. Furthermore, based on Proposition 4 , if $\alpha=2 \sqrt{2 / \pi}, \gamma=-1$ and $\beta=1$, then for any $x \in \mathbb{R}$,

$\lim _{d \rightarrow \infty} F_{P}(x ; \alpha, \beta, \gamma, d)=\Phi_{T}(x)$.

These results tell us that the following function, which we call the quasi logistic 
probability distribution function

$\Phi_{\kappa, d}(x)= \begin{cases}0, & \text { if } x \leq 0 \\ \frac{1}{1+\left(\frac{d+x}{d-x}\right)^{-\sqrt{2 / \pi} d},} & \text { if } x \in(-d, d) \\ 1, & \text { if } x \geq d\end{cases}$

can be applied to approximate the standard normal probability distribution function $\Phi(x)$, where $d>0$. Notice that $\Phi_{\kappa, d}(x)=F_{P}(x ; \alpha, \beta, \gamma, d)$ with the parameter values $\alpha=2 \sqrt{2 / \pi}, \gamma=-1$ and $\beta=1$, so the function $\Phi_{\kappa, d}(x)$ is in fact a probability distribution function.

In fuzzy logic, the linguistic modifiers like "very", "more or less", "somewhat", "rather" and "quite" over fuzzy sets that have strictly monotonously increasing or decreasing membership functions, can be modeled by the following unary operator, which is also known as the kappa function [7].

Definition 5. The kappa modifier operator (kappa function) is given by

$\kappa_{v, \nu_{0}}^{(\lambda)}(x)=\frac{1}{1+\frac{1-v_{0}}{v_{0}}\left(\frac{v}{1-v} \frac{1-x}{x}\right)^{\lambda}}$,

where $v, v_{0} \in(0,1), \lambda \in \mathbb{R}$, and $x$ is a continuous-valued logic variable.

Notice that if $v=v_{0}=0.5$, then the function $\Phi_{\kappa, d}(x)$ for $x \in(-d, d)$ can be derived from the kappa function $\kappa_{v, v_{0}}^{(\lambda)}(x)$ in (52) by setting $\lambda=\sqrt{2 / \pi} d$ and applying the $x^{\prime}=(x+d) /(2 d)$ linear transformation $(d>0)$. The $\kappa$ index in the notation $\Phi_{\kappa, d}(x)$ indicates that this function is connected with the kappa modifier operator.

It can be shown numerically that $\left|\Phi(x)-\Phi_{\kappa, d}(x)\right|$ is approximately minimal, if $d=3.1152$. In this case, the maximum absolute approximation error is $2.15 \cdot 10^{-3}$. Considering that 3.1152 is close to $\pi$, using $d=\pi$ instead of $d=3.1152$ does not worsen significantly the approximation accuracy. If $d=\pi$, then the maximum absolute approximation error is $2.3570 \cdot 10^{-3}$. Thus, we propose the use of function $\Phi_{\kappa, \pi}(x)$ for approximation as this function has a very simple formula and its maximum absolute approximation error is just slightly greater than that of function $\Phi_{\kappa, d}(x)$ with $d=3.1152[8]$ :

$\Phi_{\kappa, \pi}(x)=\left.\Phi_{\kappa, d}(x)\right|_{d=\pi}= \begin{cases}0, & \text { if } x \leq-\pi \\ \frac{1}{1+\left(\frac{\pi-x}{\pi+x}\right)^{\sqrt{2 \pi}}}, & \text { if } x \in(-\pi,+\pi) \\ 1, & \text { if } x \geq+\pi .\end{cases}$

Recall that $\Phi_{\kappa, \pi}(x)=F_{P}(x ; \alpha, \beta, \gamma, d)$ with the parameter values $\alpha=2 \sqrt{2 / \pi}$, $\beta=1, \gamma=-1$ and $d=\pi$; that is, $\Phi_{\kappa, \pi}(x)$ is a special case of the pliant probability distribution function. It should be added that there are just a few known approximations with a single constant parameter in this accuracy range (e.g. [26], [20], [2], [12]), and all these approximations include exponential terms, while $\Phi_{\kappa, \pi}(x)$ does not contain any. That is, to the best of our knowledge, in this accuracy range, there 
is no other known approximation that has such a simple formula as $\Phi_{\kappa, \pi}(x)$. The known approximations with a better accuracy have more complex formulas, while the ones with similar complex formulas do not have a higher accuracy.
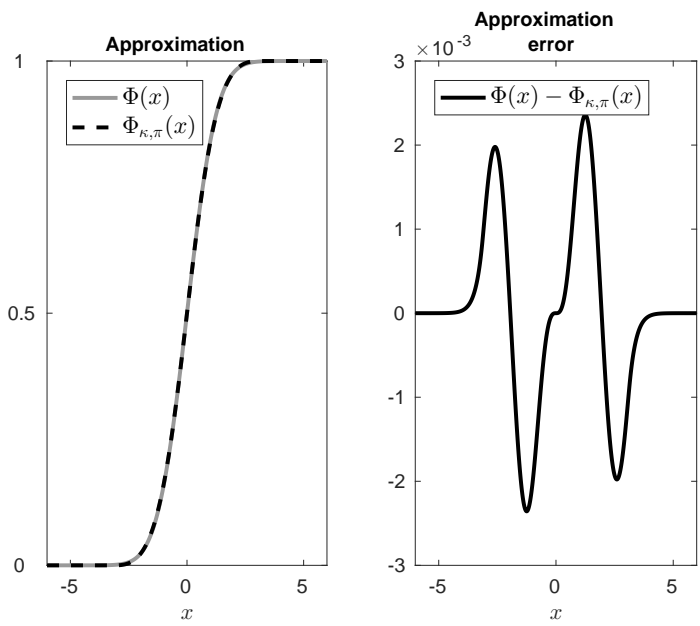

Figure 6

Approximation to the standard normal distribution function by a pliant (quasi logistic) distribution function

Figure 6 shows that the pliant probability distribution function fits quite well to the standard normal probability distribution function. Based on the above results, we can state the following proposition.

Proposition 5. If $\xi \sim D_{p}(\alpha, \beta, \gamma, d), \eta \sim N(0,1), \alpha=2 \sqrt{2 / \pi}, \gamma=-1$ and $d=\pi$ then for any $x \in \mathbb{R}$,

$\max _{x \in \mathbb{R}}|P(\eta<x)-P(\xi<x)|<2.36 \cdot 10^{-3}$.

Proof. See the previous results of this section.

\section{Conclusions}

Table 1 summarizes how the pliant probability distribution function (pliant CDF)

$F_{P}(x ; \alpha, \beta, \gamma, d)= \begin{cases}0, & \text { if } x \leq \frac{d}{2}(\gamma-1) \\ \left(1-\gamma \omega_{d}^{(-\alpha, \beta)}(x)\right)^{\gamma}, & \text { if } x \in\left(\frac{d}{2}(\gamma-1), d\right) \\ 1, & \text { if } x \geq d,\end{cases}$

where $\alpha, d \in \mathbb{R}, \alpha>0, d>0, \beta \in \mathbf{B}_{\gamma}, \gamma \in\{-1,1\}$, can be applied to approximate some well-known probability distribution functions. Based on the theoretical results and findings of our study, we may conclude that the pliant probability distribution 
Table 1

Summary of approximations by the pliant CDF

\begin{tabular}{|c|c|c|}
\hline $\begin{array}{c}\text { Parameters } \\
\text { and domain of } \\
F_{P}(x)=F_{P}(x ; \alpha, \beta, \gamma, d)\end{array}$ & $\begin{array}{l}\text { Approximated } \\
\text { CDF } \\
\end{array}$ & $\begin{array}{l}\text { Approximation } \\
\text { error }\end{array}$ \\
\hline$\alpha>0, \beta>0$ & $F_{W}(x ; \alpha, \beta)=$ & \\
\hline$\gamma=1, d>0$ & $x \leq 0$ & $\lim _{d \rightarrow \infty}\left|F_{W}(x ; \alpha, \beta)-F_{P}(x)\right|=0$ \\
\hline$x \in(0, d)$ & $1-\exp \left(-\alpha x^{\beta}\right), \quad x>0$ & \\
\hline$\alpha>0, \beta=1$ & $F_{\text {exp }}(x ; \alpha)=$ & \\
\hline$\gamma=1, d>0$ & $x \leq 0$ & $\lim _{d \rightarrow \infty}\left|F_{\text {exp }}(x ; \alpha)-F_{P}(x)\right|=0$ \\
\hline$x \in(0, d)$ & $1-\exp (-\alpha x), \quad x>0$ & \\
\hline$\alpha>0, \beta=1$ & $F_{L}(x ; \alpha)=$ & \\
\hline$\gamma=-1, d>0$ & 1 & $\lim _{d \rightarrow \infty}\left|F_{L}(x ; \alpha)-F_{P}(x)\right|=0$ \\
\hline$x \in(-d, d)$ & $\overline{1+\exp (-\alpha x)}$ & \\
\hline$\alpha=2 \sqrt{2 / \pi}, \beta=1$ & $\Phi(x)=$ & \\
\hline $\begin{array}{c}\gamma=-1, d>0 \\
x \in(-d, d)\end{array}$ & $=\frac{1}{\sqrt{2 \pi}} \int_{-\infty}^{x} \exp \left(-\frac{t^{2}}{2}\right) \mathrm{d} t$ & $\max _{x \in \mathbb{R}}\left|\Phi(x)-F_{P}(x)\right|<2.36 \cdot 10^{-3}$ \\
\hline
\end{tabular}

function may be viewed as an alternative to some key probability distribution functions including the Weibull-, exponential, logistic and standard normal probability distribution functions. We showed that our results can be utilized in a wide range of fields that include engineering, economics and the social sciences.

\section{References}

[1] S. J. Almalki and S. Nadarajah. Modifications of the Weibull distribution: A review. Reliability Engineering \& System Safety, 124:32 - 55, 2014.

[2] K. M. Aludaat and M. T. Alodat. A note on approximating the normal distribution function. Applied Mathematical Sciences, 2(9):425-429, 2008.

[3] N. Balakrishnan. Handbook of the Logistic Distribution. Statistics: A Series of Textbooks and Monographs. Taylor \& Francis, 2013.

[4] M. Bebbington, C. Lai, and R. Zitikis. A flexible Weibull extension. Reliability Engineering \& System Safety, 92(6):719 - 726, 2007.

[5] S. Bowling, M. Khasawneh, S. Kaewkuekool, and B. Cho. A logistic approximation to the cumulative normal distribution. Journal of Industrial Engineering and Management, 2(1):114-127, 2009.

[6] G. M. Cordeiro, E. M. Ortega, and S. Nadarajah. The Kumaraswamy Weibull distribution with application to failure data. Journal of the Franklin Institute, 347(8):1399 - 1429, 2010.

[7] J. Dombi. On a certain type of unary operators. In 2012 IEEE International Conference on Fuzzy Systems, pages 1-7, June 2012.

[8] J. Dombi and T. Jónás. Approximations to the normal probability distribution function using operators of continuous-valued logic. Acta Cybernetica, 23(3):829-852, 2018. 
[9] J. Dombi and T. Jónás. Kappa regression: an alternative to logistic regression. International Journal of Uncertainty, Fuzziness and Knowledge-Based Systems, accepted paper, in press, 2020.

[10] J. Dombi, T. Jónás, and Z. E. Tóth. The epsilon probability distribution and its application in reliability theory. Acta Polytechnica Hungarica, 15(1), 2018.

[11] J. Dombi, T. Jónás, Z. E. Tóth, and G. Árva. The omega probability distribution and its applications in reliability theory. Quality and Reliability Engineering International, 35(2):600-626, 2019.

[12] O. Eidous and S. Al-Salman. One-term approximation for normal distribution function. Mathematics and Statistics, 4(1):15-18, 2016.

[13] M. Ghitany, D. Al-Mutairi, N. Balakrishnan, and L. Al-Enezi. Power Lindley distribution and associated inference. Computational Statistics \& Data Analysis, 64:20 - 33, 2013.

[14] M. E. Ghitany, E. K. Al-Hussaini, and R. A. Al-Jarallah. Marshall-Olkin extended Weibull distribution and its application to censored data. Journal of Applied Statistics, 32(10):1025-1034, 2005.

[15] F. S. Hillier and G. J. Lieberman. Introduction to Operations Research, 7th Ed. McGraw-Hill, New York, USA, 2001.

[16] N. Johnson, S. Kotz, and N. Balakrishnan. Continuous univariate distributions. Number 2 in Wiley series in probability and mathematical statistics: Applied probability and statistics. Wiley \& Sons, 1995.

[17] J. Kies, N. R. L. (U.S.), and N. P. S. (U.S.). The Strength of Glass. NRL report. Naval Research Laboratory, 1958.

[18] C. D. Lai, M. Xie, and D. N. P. Murthy. A modified Weibull distribution. IEEE Transactions on Reliability, 52(1):33-37, 2003.

[19] Q. Li and H. Pham. A generalized software reliability growth model with consideration of the uncertainty of operating environments. IEEE Access, 7:84253-84267, 2019.

[20] J.-T. Lin. A simpler logistic approximation to the normal tail probability and its inverse. Applied Statistics, 39:255-257, 1990.

[21] I. Matic, R. Radoicic, and D. Stefanica. A sharp Pólya-based approximation to the normal cdf. SSRN, 2016. http://dx.doi.org/10.2139/ssrn.2842681.

[22] G. S. Mudholkar, D. K. Srivastava, and G. D. Kollia. A generalization of the Weibull distribution with application to the analysis of survival data. Journal of the American Statistical Association, 91(436):1575-1583, 1996.

[23] D. Murthy, M. Xie, and R. Jiang. Weibull Models. Wiley Series in Probability and Statistics. Wiley, 2004.

[24] A. Papoulis and S. U. Pillai. Probability, Random Variables, and Stochastic Processes. McGraw-Hill Higher Education, 4th edition, 2002. 
[25] K. K. Phani. A new modified Weibull distribution function. Journal of the American Ceramic Society, 70(8):C-182-C-184, 1987.

[26] G. Pólya. Remarks on computing the probability integral in one and two dimensions. In Proceedings of the 1st Berkeley Symposium on Mathematical Statistics and Probability, pages 63-78, Berkeley, Calif., 1949. University of California Press.

[27] A. M. Sarhan and J. Apaloo. Exponentiated modified Weibull extension distribution. Reliability Engineering \& System Safety, 112:137 - 144, 2013.

[28] G. O. Silva, E. M. M. Ortega, and G. M. Cordeiro. The beta modified Weibull distribution. Lifetime Data Analysis, 16(3):409-430, Jul 2010.

[29] K. Y. Song, I. H. Chang, and H. Pham. A software reliability model with a weibull fault detection rate function subject to operating environments. Applied Sciences, 7(10), 2017.

[30] A. Soranzo and E. Epure. Very simply explicitly invertible approximations of normal cumulative and normal quantile function. Applied Mathematical Sciences, 8(87):4323-4341, 2014.

[31] K. D. Tocher. The Art of Simulation. English University Press, London, 1963.

[32] L. Wang and Y. Shi. Reliability analysis of a class of exponential distribution under record values. Journal of Computational and Applied Mathematics, 239:367 - 379, 2013.

[33] W. Weibull. A statistical distribution function of wide applicability. Journal of Applied Mechanics, 18:293-297, 1951.

[34] M. Xie and C. Lai. Reliability analysis using an additive Weibull model with bathtub-shaped failure rate function. Reliability Engineering \& System Safety, 52(1):87 - 93, 1996.

[35] R. Yerukala and N. K. Boiroju. Approximating to the cumulative normal function and its inverse. International Journal of Scientific \& Engineering Research, 6(4):515-518, 2015.

[36] T. Yuge, M. Maruyama, and S. Yanagi. Reliability of a k-out-of-n system with common-cause failures using multivariate exponential distribution. Procedia Computer Science, 96:968 - 976, 2016.

[37] T. Zhang and M. Xie. Failure data analysis with extended Weibull distribution. Communications in Statistics - Simulation and Computation, 36(3):579-592, 2007. 